\title{
Wireless to optical phase mapping in a seamless digital wireless-photonic link
}

\author{
Niloy Ghosh, Sarang Pendharker, Member, IEEE
}

\begin{abstract}
This paper investigates the seamless mapping of wireless mmWave phase-levels to the optical phase-levels in a seamless digital mmWave-to-photonic converter. We report a non-linear mapping of phase-levels from the wireless to the optical domain which results in digital symbol degeneracy. Our work provides various optimum design guidelines to make such seamless converters practically feasible in digital communication links. The latency and the design complexity of such a seamless converter are expected to be appreciably lower than those of a conventional converter, thereby making it more suitable for 6G communication systems. Although the wireless operating frequency considered in this paper belongs to the mmWave band, all the contributions presented are equally applicable and relevant in the THz wireless band as well.
\end{abstract}

Index Terms-Wireless-photonic link, Seamless mmWave-tophotonic converter, Bi-layered modulation, EOM, TW-MZM, Digital symbol degeneracy.

\section{INTRODUCTION}

The rapidly increasing demand for ultra-high data rate, in addition to the predicted exponential surge in global traffic volume of data [1] is expected to result in congestion of the conventional frequency bands in the RF \& microwave spectrum by the end of the upcoming decade. Bearing this inevitable challenge in mind, researchers have begun exploring relatively newer frequency bands such as the mmWave and the $\mathrm{THz}$ spectrum for the revolutionary 6G wireless communication systems [2], [3]. 6G wireless communication links are expected to support ultra-high data rates owing to their high operating frequencies. However, due to challenges [4] like free-space fading, oxygen \& rain attenuation, low penetration depth, etc., the usage of high-frequency wireless communication links are limited to short-range channels in practical scenarios. This implies a tradeoff between the maximum operating wireless frequency and the maximum range of a wireless channel. A wireless-photonic link offers a potential solution to overcome this tradeoff. A high-frequency wireless signal serves a small cellular area i.e., a pico/femtocell, in a typical wireless-photonic link. A wireless-to-photonic converter located within that particular pico/femtocell receives the high-frequency wireless signal and maps it to the optical domain for transmission over a long-distance optical channel [5].

An electro-optic modulator is a fundamental unit of a wireless-to-photonic converter. A detailed analysis of a Siphotonic TW-MZM having half-power bandwidth over 30

Niloy Ghosh and Sarang Pendharker are with the Department of Electronics \& Electrical Communication Engineering, Indian Institute of Technology Kharagpur, W. Bengal, India.
$\mathrm{GHz}$ was presented in [6]. In [7], a TW series push-pull Siphotonic multi-electrode MZM was experimentally demonstrated. Si-EOMs based on free-carrier depletion were reported in [8] \& [9]. An In-P-based IQ-EOM was proposed in [10]. In [11], a monolithically integrated EOM with a CMOS-compatible drive voltage was introduced. Recently, an integrated Lithium Niobate EOM using micro-structured electrodes, overcoming voltage-bandwidth limit, has been shown in [12].

A conventional wireless-to-photonic converter usually consists of several electronic sub-blocks [13] such as low noise amplifiers, mixers, oscillators, and demodulator units, in addition to receiving antenna and electro-optic modulator unit. However, the design and integration of such electronic components at mmWave and $\mathrm{THz}$ frequencies add to the complexity of the whole system [14]-[17]. Electronic components also incorporate RC time-delay that can increase the latency of the system. Therefore, such a system may not be suitable for $6 \mathrm{G}$ applications such as virtual reality, augmented reality, medical imaging, IoT based applications, etc. [18], that necessitate the end-to-end latency period of a system to be below $1 \mathrm{~ms}$. A seamless wireless-to-photonic converter that bypasses electronic sub-blocks shall have much lower design complexity and latency than a conventional one. Such a system would be more suitable for $6 \mathrm{G}$ applications, where the electronic sub-blocks can be eliminated by directly integrating electrooptic modulators with the receiving antenna. Several antennacoupled non-linear waveguide-based electro-optic modulators have been reported in the recent literature. In [19], the design \& characterization of a polymer-based EOM integrated with a bowtie antenna was presented. The electro-optical nature of organic EO materials in plasmonic waveguides was investigated in [20]. An optical single-sideband modulator coupled with planar antennas having polarization-reversed structures was proposed for mmWave-to-photonic conversion in [21]. An InGaAs/InAlAs based MZM integrated with planar antennas was demonstrated in [22]. In these papers, the received wireless signal was considered purely sinusoidal by nature. Whereas, in modern communication systems, the information is exchanged in the form of digitally modulated signals, where the data is often encoded in the phase of the wireless signal. Therefore, it is imperative to innovate a seamless wirelessto-photonic converter that maps phase-levels of a digitally modulated wireless signal to the equivalent optical domain.

This paper primarily investigates the mapping of phase levels of an M-ary Phase Shift Keying (M-ary PSK) [23] wireless signal from the mmWave domain to the optical domain. We consider a seamless digital mmWave-to-photonic 
converter comprising of a Lithium Niobate optical channel waveguide routed under the slotted region of a centrally slotted metallic patch antenna [24]-[27]. We start Section II by discussing the bi-layered electro-optic modulation scheme used in this seamless converter. We then derive the analytical relationship between the phase-level of the received digitally modulated mmWave signal and the corresponding electrooptically modulated optical signal. We also show that the phase levels of the resultant optical signal are maximized for a specific optimum width of the patch antenna. In Section III, we report the challenge of digital symbol degeneracy, which is found to be inherent in such a seamless converter. Subsequently, we propose a potential solution to overcome this challenge by modifying the constellation diagram of the digitally modulated mmWave signal. In Section IV, we point out the dependence of the seamless phase-mapping on the freespace distance between the mmWave transmitter block and the receiving seamless converter block. We then show that this issue can be addressed by cascading multiple converter units, and we derive the optimum separation between these units. Even though the wireless frequency considered in this paper lies in the mmWave band, the key contributions of this paper are equally applicable and relevant for higher frequency bands like the THz band. This work provides important insights for practical realization of seamless digital wireless-to-photonic communication links for $6 \mathrm{G}$ applications.

\section{BI-LAYERED ELECTRO-OPTIC MODULATION IN SEAMLESS DIGITAL MMWAVE-PHOTONIC LINK}

Figure 1 compares a conventional and a seamless digital mmWave-to-photonic converter. In a conventional digital mmWave-to-photonic converter, the digital baseband signal is extracted from the mmWave carrier by demodulating the received digitally modulated mmWave signal. Then an optical carrier is modulated by the extracted digital baseband signal in the electro-optic modulator unit. The electro-optically modulated optical signal is transmitted over an optical network. Various sub-blocks of a conventional digital mmWaveto-photonic converter are shown in Fig. 1(a), wherein the digital baseband signal, the mmWave carrier, and the optical carrier have been qualitatively depicted by the green, blue and red bubbles, respectively. This scheme can be qualitatively called single-layered electro-optic modulation because a single layer of high-frequency optical carrier encapsulates the digital baseband signal in the outgoing optical signal.

Contrary to a conventional digital mmWave-to-photonic converter, a seamless converter bypasses the conventional electronic sub-units. The received digitally modulated signal directly electro-optically modulates an optical carrier, as shown in Fig. 1(b). This scheme can be called bi-layered electro-optic modulation because two layers of high-frequency carriers, i.e., a $\mathrm{GHz}$ mmWave carrier in layer-1 and a $\mathrm{THz}$ optical carrier in layer-2, encapsulate the digital baseband signal in the outgoing optical signal. In this paper we consider the received wireless mmWave signal to be M-ary PSK modulated, which can be represented as $E_{i}(t)=E_{m} \sin \left(\omega_{m w} t+a_{i}\right)$. Here $a_{i}$ represents the phase-level of $E_{i}(t)$ corresponding to the $i^{t h}$ symbol.

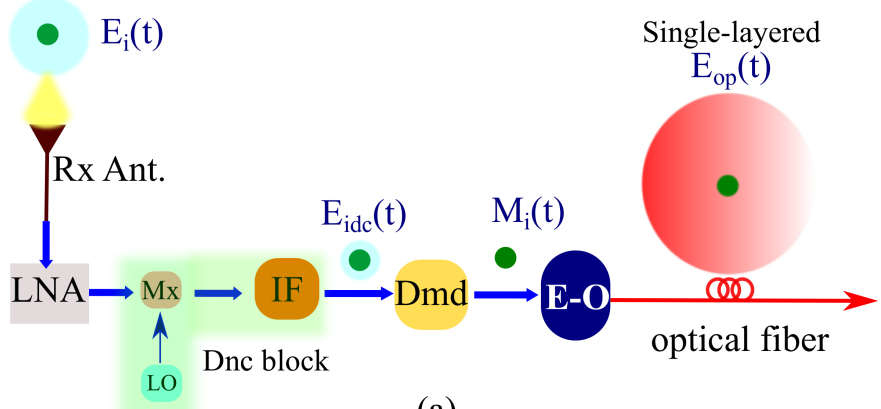

(a)

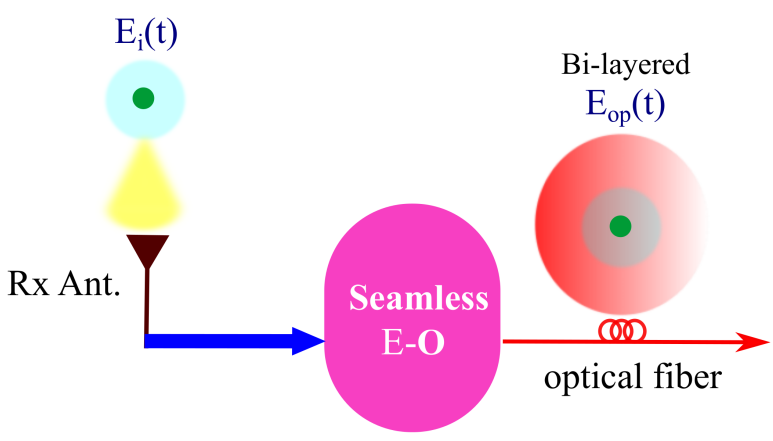

(b)

Fig. 1. Comparison between (a) Single-layered electro-optic modulation in a conventional mmWave-to-photonic converter, and (b) Bi-layered electro-optic modulation in a seamless mmWave-to-photonic converter. $E_{i}(t): i^{t h}$ symbol of the received wireless digital modulated mmWave signal, $E_{i d c}(t)$ :downconverted version of $E_{i}(t), M_{i}(t): i^{t h}$ symbol of the digital baseband signal, $E_{o p}(t)$ :bi-layered electro-optically modulated optical signal, LNA:Low Noise Amplifier, Mx:Mixer, LO:Local Oscillator, Dnc:Down-converter, IF:Intermediate-frequency Filter, Dmd:Demodulator unit, EO:Electro-Optic modulator unit, RX Ant:Receiving antenna.

We shall now investigate the analytical relationship between the phase-levels of the received M-ary PSK mmWave signal and the corresponding outgoing bi-layered modulated optical signal.

\section{A. Seamless phase-mapping from wirelss to optical}

In this paper, the seamless converter consists of a slotted metallic patch antenna integrated with a Lithium Niobate $\left(\mathrm{LiNbO}_{3}\right)$ optical channel waveguide routed under the slot gap of the antenna, as shown in Fig. 2. The metallic slotted patch antenna is designed to receive a y-polarized M-ary PSK mmWave signal of frequency $\omega_{m w}$. When the electric field intensity corresponding to the $i^{\text {th }}$ symbol of the M-ary PSK modulated mmWave signal is incident on the slotted patch antenna, a y-polarized mmWave electric field $E_{\text {slot }}(t)$, is subsequently induced in the slot gap. $E_{\text {slot }}(t)$ is essentially the enhanced version of $E_{i}(t)$ [28].

$$
E_{\text {slot }}(t)=e E_{i}(t)=e E_{m} \sin \left(\omega_{m w} t+a_{i}\right)
$$

In Eq. (1), $e$ denotes the slot-field enhancement factor. Due to the induction of $E_{\text {slot }}(t)$ in the slot gap, the section of the $\mathrm{LiNbO}_{3}$ optical waveguide located under the slotted region, gets exposed to $E_{\text {slot }}(t)$, and consequently its effective optical 


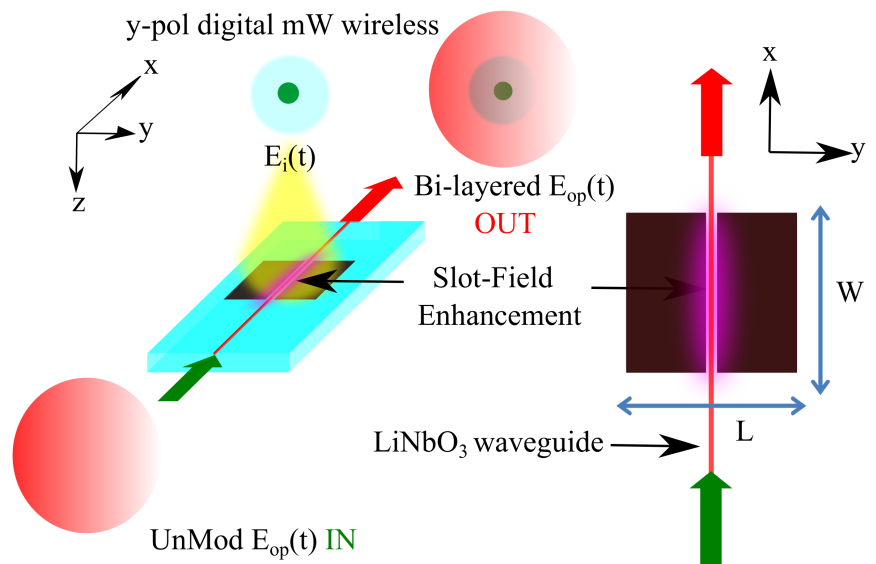

Fig. 2. The designed seamless electro-optic phase modulator's structure comprises a metallic patch antenna having dimensions $W=3.95 \mathrm{~mm} \& L=$ $3.21 \mathrm{~mm}$, with a $1 \mu \mathrm{m}$ wide slot, integrated with a $340 \mathrm{nmx} 180 \mathrm{~nm} \mathrm{LiNbO}_{3}$ optical channel waveguide routed under it.

refractive index $n_{o p}$ shifts by $\delta n_{o p}(t)$ due to Pockel's electrooptic effect [29]. The expression of $\delta n_{o p}(t)$ is governed by the following equation,

$$
\delta n_{o p}(t)=-\frac{n_{o p}^{3}}{2} r_{33} \text { e } E_{m} \sin \left(\omega_{m w} t+a_{i}\right)
$$

In Eq. (2), $r_{33}$ denotes the Pockel's electro-optic coefficient of $\mathrm{LiNbO}_{3}$ waveguide medium. Let us assume that an optical wavefront traveling within the $\mathrm{LiNbO}_{3}$ optical waveguide enters the slotted region of the patch antenna at an instant $t$. The differential phase $d \theta_{i}(t)$, accumulated by the optical wavefront due to the instantaneously varying refractive index of the waveguide medium, for a propagation over distance $d x$ through the slotted region, is given by the following equation,

$$
d \theta_{i}(t)=\beta_{o p} \delta n_{o p}(t) d x \approx \beta_{o p} \delta n_{o p}(t) \frac{c}{n_{o p}} d t
$$

In Eq. (3), $\beta_{o p}$ is the phase constant of the optical wavefront and $d t$ is the time required by the optical wavefront to travel distance $d x$ through the slotted region. In Eq. (3), the relationship between $d x$ and $d t$ has been obtained from the relation $x \approx\left(c / n_{o p}\right) t$, where $c / n_{o p}$ approximately denotes the phase velocity of the optical wavefront.

Let us consider $T$ to be the time required by the optical wavefront to travel through the entire slotted region. The length of the slotted region is equal to the width $W$ of the slotted patch antenna. By substituting the expression of $\delta n_{o p}(t)$ from Eq. (2) to Eq. (3), and by integrating the resultant equation over the duration $T$, we get the following expression of the phase accumulated by the optical wavefront corresponding to the $i^{t h}$ symbol,

$$
\theta_{i}(t)=\delta \theta E_{m} \sin \left(\omega_{m w} t+\omega_{m w} T / 2+a_{i}\right)
$$

In Eq. (4), $\delta \theta$ denotes the electro-optic modulation depth having the following expression,

$$
\delta \theta=-\frac{\beta_{o p} n_{o p}^{2} r_{33} e c}{\omega_{m w}} \sin \left(\omega_{m w} T / 2\right)
$$

It shall be shown in the upcoming subsection that the modulation depth is maximized when the patch antenna width is optimum.

\section{B. Condition for maximized modulation depth}

From Eqn. (5), it is evident that the mininum value of $T$ for which $\delta \theta$ is maximized is,

$$
T=T_{o}=\frac{\pi}{\omega_{m w}}
$$

From Eqn. (6), the optimum width $W_{o}$ of the patch antenna required for maximizing the modulation depth, can therefore be expressed as,

$$
W_{o} \approx \frac{c}{n_{o p}} T_{o}
$$

The maximum modulation depth $\delta \theta_{m}$, obtained by putting the expression of $T=T_{o}$ from Eqn. (6) into Eqn. (5), can be expressed as,

$$
\delta \theta_{m}=-\frac{\beta_{o p} n_{o p}^{2} r_{33} e c}{\omega_{m w}}
$$

Subsequently, under the optimum condition, the relationship between $a_{i}$ and $\theta_{i}(t)$ as given in Eqn. (4), reduces to the following equation,

$$
\theta_{i}(t)=\delta \theta_{m} E_{m} \cos \left(\omega_{m w} t+a_{i}\right)
$$

The expression of the bi-layered modulated optical signal $E_{o p}(t)$ having frequency $\omega_{o p}$, can therefore be given as,

$$
E_{o p}(t)=E_{o} \cos \left(\omega_{o p} t+\theta_{i}(t)\right)
$$

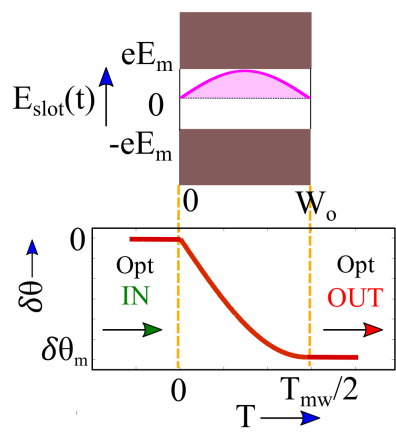

(a)

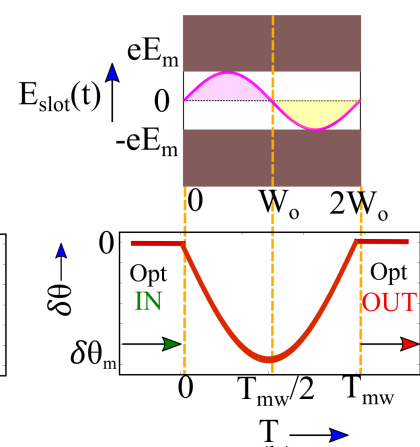

(b)
Fig. 3. Physical interpretation behind maximized modulation depth for optimum patch antenna width. (a)When the antenna width is optimum i.e. $W=W_{o}$, the net modulation depth is maximum.(b) When the antenna width is twice the optimum width i.e. $W=2 W_{o}$, the net modulation depth is zero.

The existence of optimum patch antenna width for maximized modulation depth has an important physical interpretation, as shown in Fig. 3. To travel from point $x=0$ to $W_{o}$, the 
optical wavefront takes a time that is equal to the half-cycle interval of $E_{\text {slot }}(t)$, i.e. $T=\pi /\left(\omega_{m w}\right)=T_{m w} / 2$. During this time interval, the section of the $\mathrm{LiNbO}_{3}$ waveguide located under the slotted region gets exposed only to the positive half-cycle of $E_{\text {slot }}(t)$. The modulation depth acquired by the optical wavefront in this duration is proportional to the area under the curve of $E_{\text {slot }}(t)$ in the time interval of $T=0$ to $T_{m w} / 2$. Consequently, the optical wavefront acquires a modulation depth of $\delta \theta_{m}$ by the time it reaches point $x=W_{o}$. This is shown in Fig. 3(a). When the optical wavefront travels from $x=W_{o}$ to $2 W_{o}$ i.e in the interval $T=T_{m w} / 2$ to $T_{m w}$, the section of the $\mathrm{LiNbO}_{3}$ waveguide located under the slotted region gets exposed to the negative half-cycle of $E_{\text {slot }}(t)$. As a result, optical wavefront acquires a modulation depth $\delta \theta_{m}$ of opposite polarity over and above the previously accumulated modulation depth. Therefore, the net modulation depth acquired by the optical wavefront is zero when the width of the patch antenna is $2 W_{o}$. This is shown in Fig. 3(b).

The issue of symbol degeneracy, that arises inherently in this scheme, shall be discussed in the upcoming section.

\section{SyMBOL DEGENERACY IN BI-LAYERED MODULATION}

The seamless mapping of phase-levels from the mmWave domain to the optical domain, as governed by Eq. (9) has important implications. One such implication is that of symbol degeneracy. In order to understand this, the expression of instantaneous optical phase $\theta(t)=\omega_{o p} t+\theta_{i}(t)$ from Eq. (10), must be expanded in terms of Taylor-series, as shown in the following equation,

$$
\begin{array}{r}
\theta(t)=\left(\omega_{o p}-\delta \theta_{m} E_{m} a_{i} \omega_{m w}\right) t-\delta \theta_{m} E_{m}\left(\omega_{m w}^{2} t^{2} / 2+\ldots\right) \\
+\delta \theta_{m} E_{m}\left(1-a_{i}^{2} / 2+a_{i}^{4} / 4 !+. .\right)
\end{array}
$$

The time-independent optical phase corresponding to the $i^{t h}$ symbol can therefore be expressed as,

$$
\phi_{i}=\delta \theta_{m} E_{m}\left(1-a_{i}^{2} / 2+a_{i}^{4} / 4 !+. .\right)=\delta \theta_{m} E_{m} \cos a_{i}
$$

It must be noted that there is a non-linear relationship between $a_{i}$ and $\phi_{i}$. It can further be observed that $\phi_{i}$ is an even function of $a_{i}$. This implies that any two phase-levels of equal magnitude and opposite polarity in layer-1, shall get mapped to the same phase-level in layer-2. This shall lead to erroneous phase-mapping.

This can be illustrated by considering a QPSK (4-ary PSK) modulated signal $E_{i}(t)$ in layer-1, with four symbols having phase-levels $a_{i}=\left\{45^{\circ}, 135^{\circ},-135^{\circ},-45^{\circ}\right\}$. Figure 4 shows that when the symbol state changes from ' 1 ' to ' 4 ' at $t=2 \mathrm{~ns}$, there is a $-90^{\circ}$ phase-shift in $E_{i}(t)$, as expected. However, there is no phase-shift in $E_{o p}(t)$ at $t=2 \mathrm{~ns}$. So there is no scope of tracing the symbol transition that had taken place in layer-1, just by observing the bi-layered modulated optical signal in layer-2. This implies that while symbol$1\left(a_{1}=+45^{\circ}\right)$ and symbol-4 $\left(a_{4}=-45^{\circ}\right)$ are clearly distinguishable in layer-1, they become indistinguishable in the layer-2. A similar argument can be made for symbol-2 \&

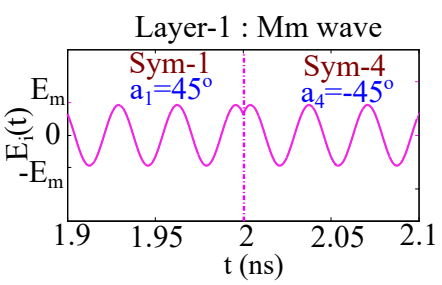

(a)

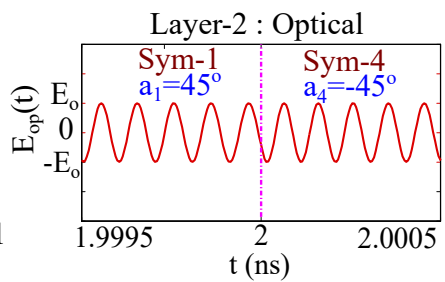

(b)
Fig. 4. (a) When the symbol state changes from ' 1 ' to ' 4 ' at $t=2 \mathrm{~ns}$, there is $-90^{\circ}$ phase-shift in $E_{i}(t)$. (b) There is no phase-shift in the corresponding bi-layered modulated optical signal $E_{o p}(t)$ at $t=2 \mathrm{~ns}$, implying that QPSK symbol-1 \& 4 become indistinguishable in layer-2.
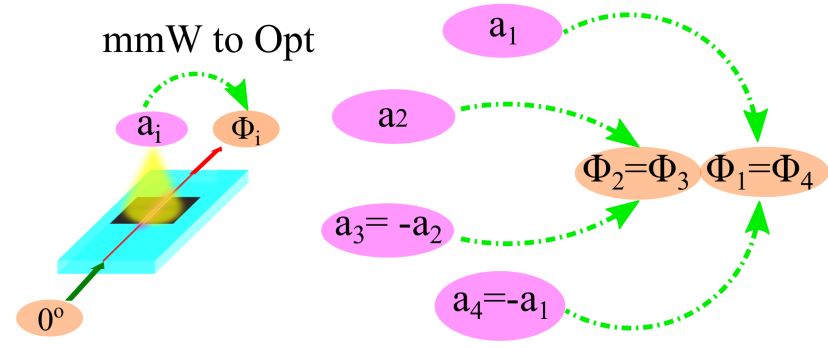

(a)

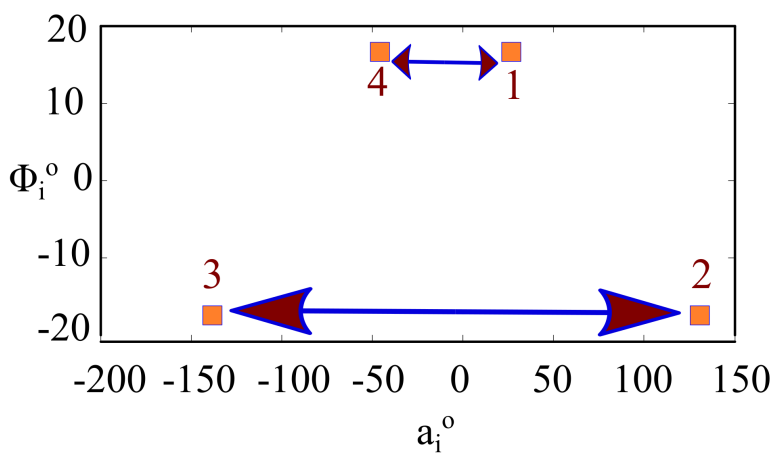

(b)

Fig. 5. (a) Depiction of digital symbol degeneracy in the proposed bi-layered modulation scheme. (b) $\phi_{i}$ vs $a_{i}$ (in degrees) for all four QPSK symbols. It can be observed that symbol-1 \& 4 in layer-1 are mapped to the same value of optical phase-level in layer-2. Similar observation can be made for symbol-2 $\& 3$.

symbol-3 of QPSK. In short, the QPSK symbol set $1 \& 4$ and set $2 \& 3$ are inherently degenerate in this conversion scheme, as depicted by Fig. 5 (a).

Figure 5(b) shows the values of $\phi_{i}$ corresponding to all four values of $a_{i}$ of QPSK. It can be seen that the value of $\phi_{i}$ is the same for $a_{i}=-45^{\circ} \&+45^{\circ}$, and for $a_{i}=-135^{\circ} \&+135^{\circ}$. For the computation of $\phi_{i}$, we have considered a patch antenna having optimum width $W_{o}=2.9 \mathrm{~mm}$, and length $L=$ $3.21 \mathrm{~mm}$, with a $1 \mu \mathrm{m}$ wide centrally located slot, deposited on a $0.3 \mathrm{~mm}$ thick substrate having dielectric constant $\epsilon_{r}=2.2$. The mmWave operating frequency is $\omega_{m w}=30 \mathrm{GHz}$. The value of slot-field enhancement factor $e$ was found to be 6485 using full-wave simulation. A 343nm x 180nm Lithium Niobate optical channel waveguide is routed under the slot gap of the designed slotted-patch antenna. The waveguide dimensions are appropriately chosen to ensure single-mode 


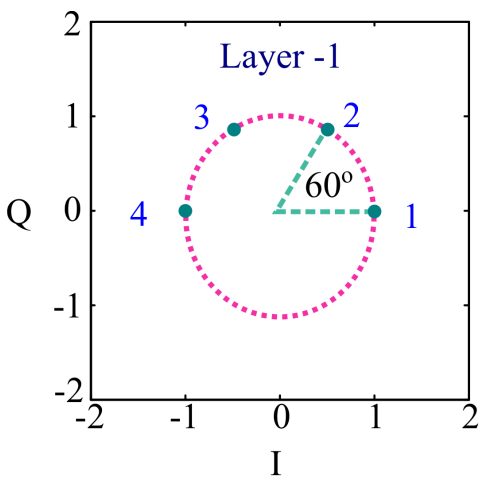

(a)

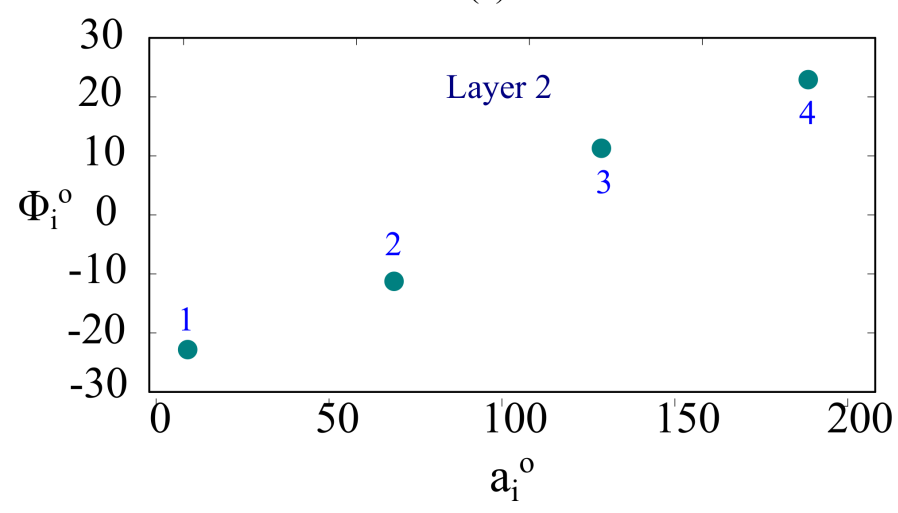

(b)

Fig. 6. (a) Optimum constellation diagram of modified 4-ary PSK in layer-1, that utilises only the upper half of the constellation diagram. (b) $\phi_{i}$ vs $a_{i}$ for the modified 4-ary PSK in layer-1.

propagation of an optical wavefront having a wavelength $1555 \mathrm{~nm}$. The value of Pockel's electro-optic coefficient of $\mathrm{LiNbO}_{3}$ is $r_{33}=31 \mathrm{pm} / \mathrm{V}$ [30] and the unperturbed effective refractive index was calculated to be $n_{o p}=1.73$. An incident electric field strength of $E_{m}=10 \mathrm{~V} / \mathrm{m}$ was considered for the computation of $\phi_{i}$ in Fig. 5(b) \& Fig. 6(b).

In a broader sense, it can be concluded that in layer- 1 constellation diagram, quadrant $1 \& 4$, and quadrant $2 \& 3$ form degenerate quadrant pairs in case of bi-layered modulation. This analysis shows that for M-ary PSK in layer-1, we can utilize either the upper half of the constellation or the lower half of the constellation to avoid symbol degeneracy in this scheme. This implies that the effective data rate would reduce by a factor of half in such a seamless conversion technique unless the constellation diagram in layer-1 is modified to address this issue. By taking the constraint of symbol degeneracy into account, an optimum constellation must be chosen so that the symbol points are located as far as possible from each other in the constellation diagram of layer-1. One such possible combination is $a_{i}=\left\{0^{\circ}, 60^{\circ}, 120^{\circ}, 180^{\circ}\right\}$. Figure 6(a) shows the constellation diagram of this modified 4-ary PSK in layer1 , in which the consecutive symbols are separated by $60^{\circ}$ and utilizes only the upper half of the constellation. Figure 6(b) shows that all four layer- 1 phase levels of the modified 4-ary PSK are mapped to four distinct layer-2 phase levels.

In the next section, we investigate the effect of free-space fading on the seamless phase-mapping.

\section{Distance DEPENDENT PHASE-MAPPING}

For reliable detection of various symbols in the optical domain, it must be made sure that the optical phase-levels corresponding to each of these symbols are separated by a particular detectable limit. However, we shall show that maintaining this optimum condition becomes a major challenge due to free-space fading.

\section{A. Effect of free-space fading on phase-mapping}

It is known from Friss's transmission equation [24] that the electric-field strength of a signal fades as it travels in free space. The relationship between the electric field strength $E_{m}$ of a mmWave signal received by the seamless converter located at a distance $R$ from a mmWave transmitter block is given by the following equation,

$$
E_{m}(R)=\frac{\sqrt{60 P_{t} G_{t}}}{R}
$$

In the above equation, $P_{t}$ is the transmitted power and $G_{t}$ is the transmitter antenna gain. We have shown previously in Eq. (12), that $\phi_{i}$ is directly proportional to $E_{m}$ as per the Pockel's electro-optic effect. Since $E_{m}$ is a function of $R$, as can be concluded from Eq. (13), it implies that $\phi_{i}$ would also be a function of $R$. Therefore, $\phi_{i}$ decays as $R$ increases, as shown in Fig. 7, which has been computed by considering $P_{t}$ to be $30 \mathrm{dBm}$ and $G_{t}$ to be $30 \mathrm{dBi}$.

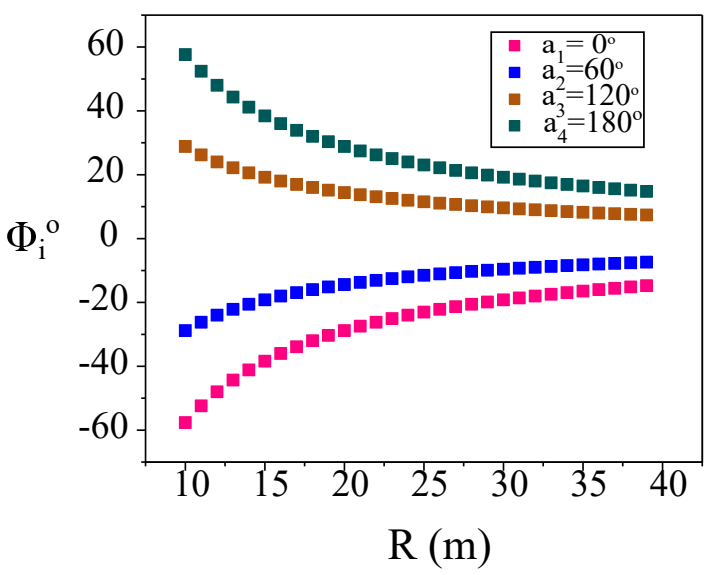

Fig. 7. Decay of layer-2 phase levels corresponding to different layer-1 symbols of modified 4-ary PSK due to free space fading.

This implies that if the distance $R$ increases, the values of $\phi_{i}$ shall become very low. Beyond a certain distance $R$, the values of $\phi_{i}$ corresponding to different symbols shall get very close to each other i.e. below the detectable limit. This would render the proposed seamless converter infeasible. There are two ways of overcoming this issue. The first way is to transmit a greater power $P_{t}$ to compensate for the greater attenuation of $E_{m}$ when $R$ is very large. However, there is often an upper limit on the transmitter power in practical cases. Another feasible option is to scale up $\phi_{i}$ by optimally cascading multiple slotted patch antenna elements in the seamless converter unit. By this method, the separation between individual optical phase-levels can be maintained 
within the detectable limit, even when the distance $R$ is very large. In the following subsection, we shall discuss the possibility of linear phase-scaling in more detail.

\section{B. Optimum separation of cascaded elements for linear phase- scaling}

In Section III, we had shown that a non-linear relationship exists between $\phi_{i}$ and the patch antenna width $W$. Therefore, $\phi_{i}$ cannot be linearly scaled up by increasing the patch antenna width. It may also appear to us at a cursory glance that $\phi_{i}$ can be scaled up by simply cascading multiple slotted patch antennas to form an array. However, we have found that arbitrary cascading of antenna elements does not ensure linear scaling of $\phi_{i}$. The periodicity of the cascaded elements plays an important role in this case. For better visualization of this argument, it is important to derive the expression of the net optical phase acquired by an optical wavefront as it propagates through the $\mathrm{LiNbO}_{3}$ optical waveguide routed under $\mathrm{N}$ number of cascaded slotted patch antenna elements, each having optimum width $W_{o}$. Firstly, we integrate Eq. (3) from time instant $\left(t+m n_{o p} W_{p} / c\right)$ to time instant $\left(t+m n_{o p} W_{p} / c+T_{o}\right)$, for $m=0,1,2,3 \ldots .(N-1)$. Here $W_{p}$ denotes the element periodicity of the array. The summation of all the integrals corresponding to all the values of $m$ gives the net phase accumulated by the optical wavefront, as shown in the following equation,

$\theta_{i}(t, N)=\delta \theta_{N} E_{m} \cos \left(\omega_{m w} t+a_{i}+(N-1) \omega_{m w} \frac{n_{o p}}{2 c} W_{p}\right)$

In the above equation, $\delta \theta_{N}$ represents the scaled modulation depth, which can be expressed as,

$$
\delta \theta_{N}=\delta \theta_{m} \frac{\sin \left(N \omega_{m w} W_{p} n_{o p} / 2 c\right)}{\sin \left(\omega_{m w} W_{p} n_{o p} / 2 c\right)}
$$

From Eq. (15) it can observed that $\delta \theta_{N}$ is maximized when $W_{p}=2 \pi c / \omega_{m w} n_{o p}=2 c T_{o} / n_{o p}=2 W_{o}$. For this optimum value of $W_{p}, \delta \theta_{N}$ scales linearly with $N$, as shown by the following equation,

$$
\delta \theta_{N \max }=N \delta \theta_{m}
$$

From Eq. (16), it can be concluded that the linear scaling of modulation depth takes place by increasing the number of cascaded elements only when the element periodicity is twice the optimum width of the individual patch antennas. Figure 8 (a) corroborates this argument, which clearly shows that $\left|\delta \theta_{N}\right|$ increases linearly with $\mathrm{N}$ when $W_{p}=2 W_{o}$. However, it can also be seen that $\left|\delta \theta_{N}\right|$ is not a linear function of $\mathrm{N}$ when $W_{p}=W_{o}$. Figure 8(b) shows that the optical phase levels corresponding to different symbols get scaled up linearly as the number of optimally spaced antenna elements are increased. The physical interpretation of this argument has been illustrated in Fig. 9, which shows a 3-element optimally cascaded patch antenna elements, each having optimum width. When an optical wavefront leaves the first antenna element at $T=T_{m w} / 2$, it would have already acquired a modulation

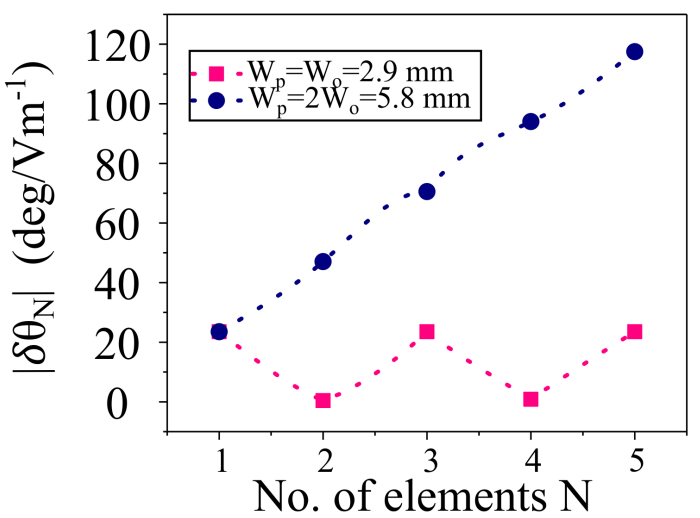

(a)

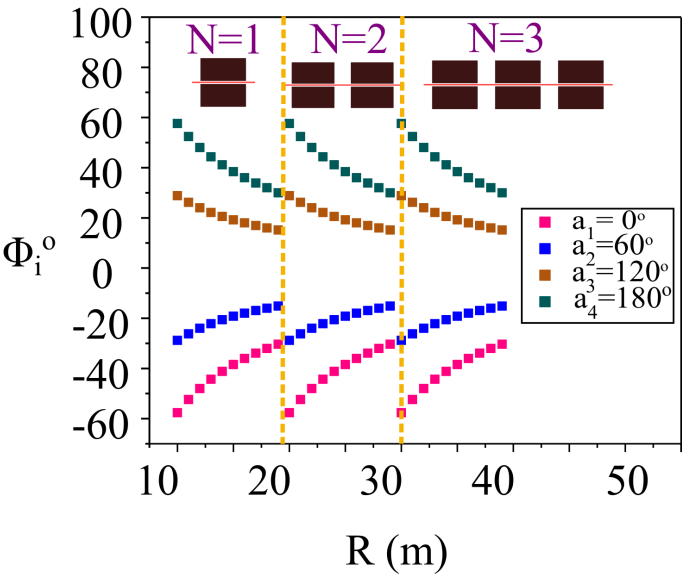

(b)

Fig. 8. (a) Comparison of the nature of modulation depth scaling when the element periodicity is $W_{o}$ and $2 W_{o}$, respectively. (b) Linear scaling up of optical phase levels corresponding to different symbols of modified 4-ary PSK.

depth of $\delta \theta_{m}$, as we had seen earlier in Fig. 3. Then the optical wavefront travels between the exit point of the first element and entry point of the second element. The spacing between the exit and entry of the first and second element respectively is $W_{o}$. This means that $E_{\text {slot }}(t)$ goes over its negative cycle as the optical wavefront travels between the first and second elements. The section of the $\mathrm{LiNbO}_{3}$ located under the spacing between two consecutive elements does not get exposed to the negative cycle of $E_{\text {slot }}(t)$. Due to this, the modulation depth $\delta \theta_{m}$, that was previously accumulated by the optical wavefront does not get perturbed when it travels between the spacing between the first and the second antenna elements. By the time the optical wavefront reaches point $x=2 W_{o}$ at $T=T_{m w}$, $E_{\text {slot }}(t)$ would have already finished a complete cycle. So, when the optical wavefront travels through the second element having width $W_{o}$, it is again only exposed to the positive cycle of $E_{\text {slot }}(t)$, resulting in the accumulation of modulation depth $\delta \theta_{m}$ over and above the previously accumulated modulation depth $\delta \theta_{m}$. Therefore, the net modulation depth acquired by it when it leaves the second element is $2 \delta \theta_{m}$. Similarly, the net modulation depth acquired by it when it leaves the 
third element is $3 \delta \theta_{m}$, and so on. This way, by cascading $\mathrm{N}$ elements with optimum width $W_{o}$ and with element periodicity $2 W_{o}$, the modulation depth can be scaled up by a factor of $\mathrm{N}$.

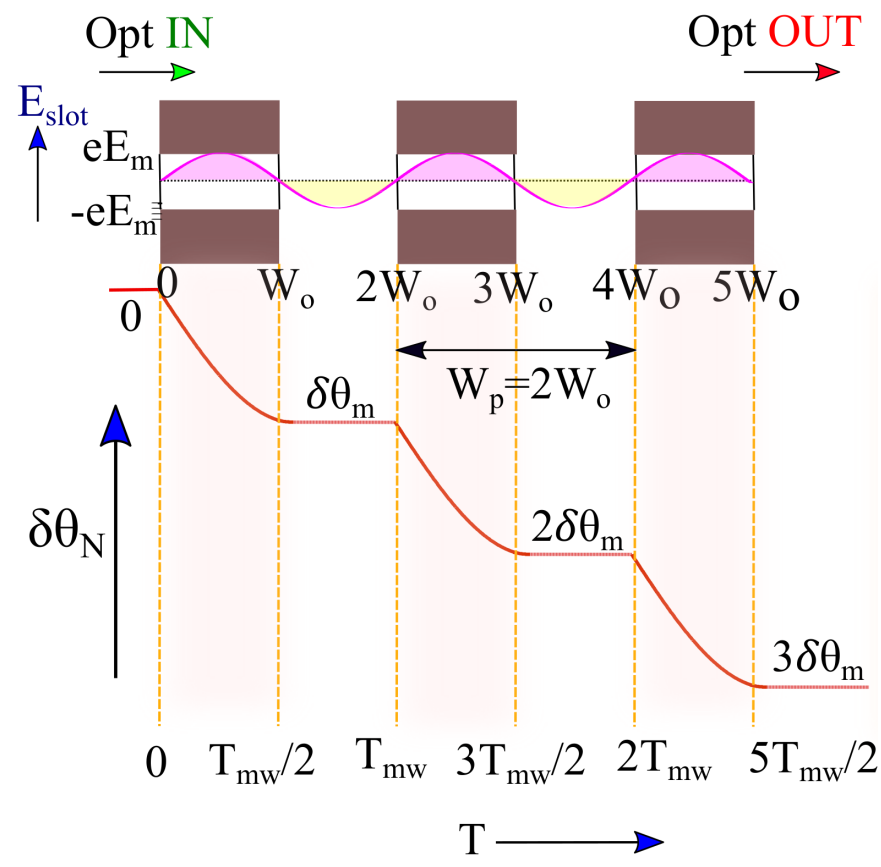

Fig. 9. Physical interpretation behind linear scaling up of modulation depth by cascading multiple optimally spaced patch antenna elements.

\section{CONCLUSiON}

This paper discusses the seamless mapping of phase-levels of a digitally modulated signal from the mmWave domain to the optical domain. We have derived the analytical relationship between the phase levels of the M-ary PSK wireless signal and the bi-layered modulated optical signal. We have investigated digital symbol degeneracy that arises inherently in this seamless conversion scheme and reported modifications required in the wireless M-ary PSK constellation diagram. We further show that seamless phase mapping is significantly affected by free-space fading. The design guidelines for mitigation of distant dependent phase-mapping have also been presented. The design principles reported in this work are necessary for implementing seamless wireless-to-photonic links in $6 \mathrm{G}$ networks.

\section{ACKNOWLEDGMENT}

Authors would like to thank Dr. Amitalok J. Budkuley for the technical insights on digital modulation schemes and constellation diagrams. Authors would also like to acknowledge the ISIRD funding (code: PRD) from IIT Kharagpur.

\section{REFERENCES}

[1] I. Union, "IMT M.2370-0 traffic estimates for the years 2020 to 2030," Report ITU, 2015.

[2] I. Tomkos, D. Klonidis, E. Pikasis, and S. Theodoridis, "Toward the 6G Network Era: Opportunities and Challenges," IT Professional, vol. 22, no. 1 , pp. $34-38,2020$.
[3] M. Z. Chowdhury, M. Shahjalal, S. Ahmed, and Y. M. Jang, "6G Wireless Communication Systems: Applications, Requirements, Technologies, Challenges, and Research Directions," IEEE Open Journal of the Communications Society, vol. 1, pp. 957-975, 2020.

[4] T. Rappaport, Wireless Communications: Principles and Practice, 2nd ed. USA: Prentice Hall PTR, 2001.

[5] X. N. Fernando, Radio over fiber for wireless communications: From fundamentals to advanced topics. John Wiley \& Sons, 2014.

[6] A. Samani, M. Chagnon, D. Patel, V. Veerasubramanian, S. Ghosh, M. Osman, Q. Zhong, and D. V. Plant, "A Low-Voltage 35-GHz Silicon Photonic Modulator-Enabled 112-Gb/s Transmission System," IEEE Photonics Journal, vol. 7, no. 3, pp. 1-13, 2015.

[7] A. Samani, D. Patel, M. Chagnon, E. El-Fiky, R. Li, M. Jacques, N. Abadía, V. Veerasubramanian, and D. V. Plant, "Experimental parametric study of $128 \mathrm{~Gb} / \mathrm{s}$ PAM-4 transmission system using a multielectrode silicon photonic Mach Zehnder modulator," Optics express, vol. 25, no. 12, pp. 13252-13 262, 2017.

[8] D. J. Thomson, F. Y. Gardes, J.-M. Fedeli, S. Zlatanovic, Y. Hu, B. P. P. Kuo, E. Myslivets, N. Alic, S. Radic, G. Z. Mashanovich, and G. T. Reed, "50-Gb/s Silicon Optical Modulator," IEEE Photonics Technology Letters, vol. 24, no. 4, pp. 234-236, 2012.

[9] D. J. Thomson, F. Y. Gardes, G. T. Reed, F. Milesi, and J.-M. Fedeli, "High speed silicon optical modulator with self aligned fabrication process," Opt. Express, vol. 18, pp. 19 064-19 069, Aug 2010.

[10] Y. Ogiso, J. Ozaki, Y. Ueda, N. Kashio, N. Kikuchi, E. Yamada, H. Tanobe, S. Kanazawa, H. Yamazaki, Y. Ohiso, et al., "Over 67 $\mathrm{GHz}$ bandwidth and $1.5 \mathrm{~V} V_{\pi}$ InP-based optical IQ modulator with nipn heterostructure," Journal of lightwave technology, vol. 35, no. 8, pp. 1450-1455, 2016.

[11] C. Wang, M. Zhang, X. Chen, M. Bertrand, A. Shams-Ansari, S. Chandrasekhar, P. Winzer, and M. Lončar, "Integrated Lithium Niobate electro-optic modulators operating at CMOS-compatible voltages," $\mathrm{Na}$ ture, vol. 562, no. 7725, pp. 101-104, 2018.

[12] P. Kharel, C. Reimer, K. Luke, L. He, and M. Zhang, "Breaking voltagebandwidth limits in integrated Lithium Niobate modulators using microstructured electrodes," Optica, vol. 8, no. 3, pp. 357-363, 2021.

[13] D. M. Pozar, Microwave Engineering. John wiley \& sons, 2011.

[14] A. Hamani, A. Siligaris, B. Blampey, C. Dehos, and J. L. Gonzalez Jimenez, "A 125.5-157 GHz $8 \mathrm{~dB}$ NF and $16 \mathrm{~dB}$ of Gain D-band Low Noise Amplifier in CMOS SOI 45 nm," in 2020 IEEE/MTT-S International Microwave Symposium (IMS), 2020, pp. 197-200.

[15] R. A. Shaheen, T. Rahkonen, and A. Pärssinen, "Mmwave Frequency Reconfigurable Low Noise Amplifiers for 5G," IEEE Transactions on Circuits and Systems II: Express Briefs, 2020.

[16] A. Ahmed, M.-Y. Huang, and H. Wang, "Mixer-first extremely wideband 43-97GHz RX frontend with broadband quadrature input matching and current mode transformer-based image rejection for massive MIMO applications," in 2020 IEEE Custom Integrated Circuits Conference (CICC). IEEE, 2020, pp. 1-4.

[17] X. Lu, C. R. Chappidi, X. Wu, and K. Sengupta, "Antenna preprocessing and element-pattern shaping for multi-band mmwave arrays: Multi-port receivers and antennas," IEEE Journal of Solid-State Circuits, vol. 55, no. 6, pp. 1455-1470, 2020.

[18] P. Yang, Y. Xiao, M. Xiao, and S. Li, "6G wireless communications: Vision and potential techniques," IEEE Network, vol. 33, no. 4, pp. 7075, 2019.

[19] O. D. Herrera, K.-J. Kim, R. Voorakaranam, R. Himmelhuber, S. Wang, V. Demir, Q. Zhan, L. Li, R. A. Norwood, R. L. Nelson, et al., "Silica/electro-optic polymer optical modulator with integrated antenna for microwave receiving," Journal of Lightwave Technology, vol. 32, no. 20, pp. 3861-3867, 2014.

[20] W. Heni, C. Haffner, D. L. Elder, A. F. Tillack, Y. Fedoryshyn, R. Cottier, Y. Salamin, C. Hoessbacher, U. Koch, B. Cheng, et al., "Nonlinearities of organic electro-optic materials in nanoscale slots and implications for the optimum modulator design," Optics express, vol. 25, no. 3, pp. 2627-2653, 2017.

[21] Y. Matsukawa, I. Toshiyuki, H. Murata, and A. Sanada, "Millimeterwave-band optical single-sideband modulator using array-antennaelectrode and polarization-reversed structures," in 2017 Opto-Electronics and Communications Conference (OECC) and Photonics Global Conference $(P G C)$. IEEE, 2017, pp. 1-5.

[22] Y. Miyazeki and T. Arakawa, "Proposal of InGaAs/InAlAs multiple quantum well Mach-Zehnder modulator integrated with array of planar antennas," Japanese Journal of Applied Physics, vol. 58, no. SJ, p. SJJE05, 2019

[23] S. Haykin, Communication systems. John Wiley \& Sons, 2008. 
[24] C. A. Balanis, Antenna theory: analysis and design. John wiley \& sons, 2015.

[25] G. Kumar and K. P. Ray, Broadband microstrip antennas. Artech house, 2003.

[26] R. Bancroft, Microstrip and printed antenna design. The Institution of Engineering and Technology, 2009.

[27] R. Garg, P. Bhartia, I. J. Bahl, and A. Ittipiboon, Microstrip antenna design handbook. Artech house, 2001.

[28] Y. N. Wijayanto, H. Murata, and Y. Okamura, "Electrooptic MillimeterWave-Lightwave Signal Converters Suspended to Gap-Embedded Patch Antennas on Low- $k$ Dielectric Materials," IEEE Journal of Selected Topics in Quantum Electronics, vol. 19, no. 6, pp. 33-41, 2013.

[29] D. Marcuse, Principles of quantum electronics. Elsevier, 2012.

[30] K. Wong, Properties of lithium niobate, ser. EMIS datareview series; no. 28. IEE, 2002. 